\title{
L'aferesi terapeutica nella miastenia grave
}

\author{
Carlo Antozzi \\ U.O. Neurologia IV - Neuroimmunologia e Patologia Muscolare, Fondazione IRCCS Istituto Neurologico C. Besta, Milano
}

\begin{abstract}
THERAPEUTIC APHERESIS IN MYASTHENIA GRAVIS
Abstract. Plasmapheresis has considerably improved the management of patients affected by myasthenia gravis (MG), particularly in case of severe acute worsening with bulbar impairment. Controlled trials against placebo are not available in this field, but evidences emerged from open-label studies are considered a strong confirmation of the clinical efficacy of plasmapheresis in MG. More recently, the techniques of selective apheresis based on the properties of protein A and sheep anti-human IgG have been introduced into clinical practice, allowing chronic apheresis in treatment-resistant MG patients.
\end{abstract}

Key words: Plasmapheresis, Myasthenia gravis, Autoimmunity

Conflict of interest: Travels and congress expenses from Merck, Teva and Fresenius Medical Care

Financial Support: None.

Ricevuto: 8 Febbraio 2013; Accettato: 21 Marzo 2013

\section{Introduzione}

Dalla prima segnalazione risalente al 1976 da parte di Pinching e Newsom-Davis (1), l'aferesi terapeutica rappresenta uno strumento terapeutico cruciale nel trattamento della miastenia grave (MG). Nel corso degli ultimi due decenni sono stati fatti considerevoli progressi sul piano tecnologico nel campo del trattamento plasmatico della malattie autoimmuni. Pur in assenza di studi controllati e di una precisa standardizzazione dei protocolli, la MG è la candidata ideale al trattamento aferetico, in quanto conosciamo i meccanismi patogenetici alla base della malattia: infatti, sono stati identificati due autoanticorpi specifici diretti rispettivamente contro il recettore acetilcolinico (AChR-Ab) o la tirosin-kinasi muscolo specifica (MuSK-Ab), i due bersagli antigenici localizzati a livello della giunzione neuromuscolare coinvolti nella malattia (2). La plasmaferesi è stata introdotta come presidio terapeutico riservato soprattutto a pazienti in condizioni di peggioramento acuto; inoltre, i miglioramenti a livello tecnologico hanno considerevolmente migliorato la tollerabilità dello scambio plasmatico e l'introduzione delle tecniche di rimozione selettiva degli anticorpi ha reso possibile il trattamento a lungo termine dei pazienti farmaco-resistenti.

\section{L'aferesi terapeutica nella miastenia}

Nel 1986, una Consensus Conference organizzata dal NIH sull'uso della plasmaferesi nella MG ha concluso che la procedura è efficace a breve termine e che uno studio controllato contro placebo non è realizzabile ed eticamente non è giustificato $(3,4)$. Di conseguenza, non disponiamo di studi basati sull'evidenza e di Linee Guida che rispondano a tali criteri. Gli aspetti metodologici relativi all'eterogeneità dei lavori osservazionali sono stati sottolineati nella review Cochrane dedicata all'argomento (5). L'unico studio controllato disponibile prevedeva il confronto della plasmaferesi con le immunoglobuline endovenose, studio che ha evidenziato l'equivalenza delle due terapie (6).

Nonostante l'eterogeneità, gli studi aperti riportati in letteratura hanno mostrato che la plasmaferesi è efficace nel 60-70\% dei pazienti trattati e che il miglioramento clinico è temporalmente in relazione con la rimozione plasmatica. L'eterogeneità dei protocolli di trattamento descritti è molto ampia e non disponiamo di un'indicazione precisa sul numero ottimale di sedute da effettuare; la media delle esperienze riportate in letteratura varia da un minimo di tre a un massimo di sei sedute aferetiche con rimozione di 1-1.5 volumi plasmatici teorici, preferibilmente effettuate a giorni alterni. La nostra esperienza ha evidenziato che anche un protocollo breve di due sedute a giorni alterni è efficace nel $70 \%$ dei pazienti trattati (7).

\section{Indicazioni allo scambio plasmatico nella MG}

L'indicazione principale è il peggioramento acuto della malattia (MG grave generalizzata o bulbare) o nella crisi miastenica. Altre indicazioni includono il peggioramento all'inizio della terapia steroidea e nella preparazione alla timectomia nei pazienti sintomatici. Sulla base della nostra esperienza riteniamo che non vi sia alcuna necessità di effettuare la plasmaferesi immediatamente prima della timectomia, quando la malattia è ben controllata dalla terapia farmacologica. L'uso cronico della plasmaferesi non è stato affrontato con un protocollo 
preciso. Tuttavia, la plasmaferesi può essere usata a intervalli ripetuti in pazienti selezionati in caso di recidive frequenti, quando la risposta alla terapia è insoddisfacente dopo un adeguato follow-up clinico o in pazienti con controindicazioni all'uso dei corticosteroidi ad alte dosi. Limitazioni all'uso cronico sono rappresentate dalla necessità di accessi vascolari adeguati e dagli ovvi effetti sui diversi componenti del plasma in caso di protocolli intensivi. Pertanto, il numero di sessioni e l'intervallo tra loro devono essere adattati ad ogni singolo paziente. A causa delle suddette limitazioni, è possibile prendere in considerazione l'aferesi selettiva come tecnica di scelta in pazienti con forme scompensate e farmacoresistenti, con necessità di trattamenti intensivi e prolungati.

\section{La plasmaferesi e la MG associata ad anticorpi antiMuSK}

Questi pazienti possono essere distinti dai pazienti con anticorpi anti-AChR per il tipico coinvolgimento "oculo-bulbare" e per l'incidenza più frequente di compromissione respiratoria e crisi miastenica. La malattia in questi pazienti può, almeno nella fase iniziale, presentare una risposta meno marcata alle tradizionali terapie immunosoppressive, ma questi pazienti presentano una risposta molto rapida alla plasmaferesi, caratteristica molto importante, data l'elevata incidenza di disturbi bulbari e di difficoltà respiratorie in questo sottogruppo di pazienti miastenici (8).

\section{L'aferesi selettiva nella MG}

L'approccio aferetico ideale dovrebbe rimuovere esclusivamente gli autoanticorpi specifici coinvolti nella patogenesi della malattia in trattamento, lasciando tutti gli altri componenti del plasma inalterati. Un approccio così specifico non è ancora disponibile nella pratica clinica. Un compromesso è rappresentato dalla rimozione selettiva delle immunoglobuline $\mathrm{G}$ ( $\mathrm{IgG}$ ) circolanti mediante immunoassorbimento (IA). Due leganti diversi sono disponibili per l'uso clinico nelle patologie anticorpo-mediate. Il primo è rappresentato dalla proteina $\mathrm{A}$, un componente della parete cellulare dello stafilococco e ha la particolarità di legare le IgG umane con alta affinità; si ritiene che il legame sia mediato dal frammento Fc delle IgG. Un secondo metodo prevede l'utilizzo di anticorpi policlonali di pecora antiIgG umane, trattandosi, in questo caso, di un legame di tipo immunologico (9). In entrambi i casi, il plasma deve essere separato dalla parte corpuscolata e, quindi, viene passato on-line attraverso una serie di due filtri contenenti il legante delle IgG. I filtri sono gestiti da un monitor dedicato e lavorano alternativamente: mentre il primo filtro rimuove le IgG, il secondo viene lavato, sottoposto al processo di eluizione per rimuovere le IgG adsorbite e, infine, riempito con una soluzione tampone, pronto per il ciclo successivo di adsorbimento. Queste caratteristiche rendono la tecnica adatta al trattamento di una quantità illimitata di plasma, in quanto non è necessario alcun liquido sostitutivo e l'interazione con i fattori della coagulazione è trascurabile. Il nostro protocollo di trattamento consiste in tre sedute con trattamento di almeno due volumi di plasma ciascuna, eseguite a giorni alterni.

In caso di necessità, possono essere effettuati anche protocolli più intensivi in condizioni di sicurezza, in quanto non è richiesto alcun fluido sostitutivo. Dopo il primo ciclo ripetiamo una-due sedute di mantenimento ogni quattro settimane; quando il miglioramento clinico si mantiene stabile tra sessioni consecutive e l'intervallo tra le stesse supera $\mathrm{i}$ due mesi senza una ricaduta clinica, si può prendere in considerazione la sospensione del trattamento selettivo $(10,11)$. Si consiglia l'utilizzo dell'IA in pazienti con MG resistente al trattamento dopo un adeguato follow-up clinico, in pazienti che richiedono frequenti plasmaferesi per mantenere una condizione clinica soddisfacente o con controindicazioni all'uso di corticosteroidi ad alto dosaggio o di farmaci immunosoppressori. Non esistono protocolli validati o condivisi ma le casistiche riportate in letteratura confermano l'utilità dell'IA nel trattamento cronico $(12,13)$.

Un'ulteriore metodica è rappresentata dalle colonne di triptofano legato covalentemente al polivinil-alcool utilizzato nella MG con promettenti risultati iniziali in termini di miglioramento clinico $(14,15)$. Il legame è mediato da un'interazione di tipo chimico ed è meno selettivo rispetto alla proteina A o alle IgG di pecora anti-IgG umane.

\section{Conclusioni}

L'introduzione e il progressivo miglioramento tecnologico delle metodiche aferetiche hanno modificato radicalmente la gestione della MG specialmente nella fase acuta e, in particolare, nella gestione del paziente con deficit bulbare. In parallelo, la disponibilità dell'aferesi selettiva si è rivelata particolarmente utile a lungo termine, consentendo un'immunomodulazione altrimenti non facilmente ottenibile con la plasmaferesi tradizionale. Dal punto di vista teorico, è emerso il razionale di poter disporre di un'aferesi non solo selettiva ma anche specifica, approccio realizzato in vitro mediante frammenti ricombinanti del recettore acetilcolinico, con interessanti risultati preliminari. $\mathrm{Ci}$ auguriamo che questo nuovo approccio possa raggiungere la fase di sperimentazione nell'uomo (16).

\section{Riassunto}

La plasmaferesi terapeutica ha migliorato considerevolmente il trattamento dei pazienti affetti da miastenia grave (MG), in particolare nei casi con deterioramento acuto del quadro clinico con deficit bulbare. Non sono disponibili studi controllati in questo campo, ma le esperienze emerse dagli studi aperti sostengono positivamente l'utilizzo dell'aferesi terapeutica nella MG. Più recentemente sono state introdotte le tecniche aferetiche semiselettive adatte al trattamento cronico dei pazienti farmaco-resistenti.

Parole chiave: Plasmaferesi, Miastenia grave, Malattie autoimmuni 
Dichiarazione di conflitto di interessi: Spese congressuali sostenute da Merck, Teva and Fresenius Medical Care.

Contributi economici degli autori: L'Autore dichiara di non aver ricevuto sponsorizzazioni economiche per la preparazione dell'articolo.

Indirizzo degli Autori:

Dr. Carlo Antozzi

U.O. Neurologia IV - Neuroimmunologia e

Patologia Muscolare

Fondazione IRCCS Istituto Neurologico C. Besta

Via Celoria 11

20133 Milano

antozzi@istituto-besta.it

\section{Bibliografia}

1. Pinching AJ, Peters DK. Remission of myasthenia gravis following plasma-exchange. Lancet 1976; 2: 1373-6.

2. Cavalcante P, Bernasconi P, Mantegazza R. Autoimmune mechanisms in myasthenia gravis. Curr Opin Neurol 2012; 25 : 621-9.

3. The utility of therapeutic plasmapheresis for neurological disorders. Natl Inst Health Consens Dev Conf Consens Statement 1986: 6: 1-7.

4. Assessment of plasmapheresis. Report of the Therapeutics and Technology Assessment Subcommittee of the American Academy of Neurology. Neurology 1996; 47: 840-3.
5. Gajdos P, Chevret S, Toyka K. Plasma exchange for myasthenia gravis. Cochrane Database Syst Rev 2002; (4): CD002275.

6. Gajdos P, Chevret S, Clair B, et al. Clinical trial of plasma exchange and high dose immunoglobulin in myasthenia gravis. Ann Neurol 1997; 41: 789-96.

7. Antozzi C, Gemma M, Regi B, et al. A short plasma exchange protocol is effective in severe myasthenia gravis. J Neurol 1991; 238: 103-7.

8. Baggi F, Andreetta A, Maggi L, et al. Complete stable remission and autoantibody specificity in myasthenia gravis. Neurology 2013; 80: 188-95.

9. Bosch T. Therapeutic apheresis. State of the art in the year 2005 . Ther Apher Dial 2005; 9: 459-68.

10. Antozzi C, Berta E, Confalonieri P, et al. Protein-A immunoadsorption in immunosuppression-resistant myasthenia gravis. Lancet 1994; 343: 124.

11. Berta E, Confalonieri P, Simoncini O, et al. Removal of anticetylcholine receptor antibodies by protein-A immunoadsorption in myasthenia gravis. Int J Artif Organs 1994; 17: 603-8.

12. Benny WB, Sutton DM, Oger J, Bril V, McAteer MJ, Rock G. Clinical evaluation of a staphylococcal protein A immunoadsorption system in the treatment of myasthenia gravis. Transfusion 1999; 39: 682-7.

13. Haas M, Mayr N, Zeitholfer J, et al. Long-term treatment of myasthenia gravis with immunoadsorption. J Clin Apher 2002; 17: 84-7.

14. Shibuya N, Sato T, Osame M, et al. Immunoadsorption therapy for myasthenia gravis. J Neurol Neurosurg Psych 1994; 57: 578-81.

15. Grob D, Simpson D, Mitsumoto H, et al. Treatment of myasthenia gravis by immunoadsorption of plasma. Neurology 1995; $45 ; 338-44$

16. Zisimopoulou P, Lagoumintzis G, Kostelidou K, et al. Towards antigen-specific apheresis of pathogenic autoantibodies in the treatment of myasthenia gravis by plasmapheresis. J Neuroimmunol 2008; 201-202: 95-103. 